\title{
ARTICLE
}

Epidemiology

\section{A systematic review of meta-analyses assessing the validity of tumour response endpoints as surrogates for progression-free or overall survival in cancer}

\author{
Katy Cooper $\mathbb{I}^{1}$, Paul Tappenden $\mathbb{D D}^{1}$, Anna Cantrell $\mathbb{D}^{1}$ and Kate Ennis $\mathbb{( D}^{1}$
}

BACKGROUND: Tumour response endpoints, such as overall response rate (ORR) and complete response (CR), are increasingly used in cancer trials. However, the validity of response-based surrogates is unclear. This systematic review summarises meta-analyses assessing the association between response-based outcomes and overall survival (OS), progression-free survival (PFS) or time-toprogression (TTP).

METHODS: Five databases were searched to March 2019. Meta-analyses reporting correlation or regression between responsebased outcomes and OS, PFS or TTP were summarised.

RESULTS: The systematic review included 63 studies across 20 cancer types, most commonly non-small cell lung cancer (NSCLC), colorectal cancer (CRC) and breast cancer. The strength of association between ORR or CR and either PFS or OS varied widely between and within studies, with no clear pattern by cancer type. The association between ORR and OS appeared weaker and more variable than that between ORR and PFS, both for associations between absolute endpoints and associations between treatment effects.

CONCLUSIONS: This systematic review suggests that response-based endpoints, such as ORR and CR, may not be reliable surrogates for PFS or OS. Where it is necessary to use tumour response to predict treatment effects on survival outcomes, it is important to fully reflect all statistical uncertainty in the surrogate relationship.

British Journal of Cancer (2020) 123:1686-1696; https://doi.org/10.1038/s41416-020-01050-w

\section{BACKGROUND}

Decisions about the use of new and existing health technologies should ideally be informed by estimates of treatment effects derived from high-quality randomised controlled trials (RCTs), which measure patient-relevant endpoints over a clinically appropriate timeframe. Such "final" endpoints typically involve the measurement of health benefits, which reflect aspects of the disease, and its treatment, which are important to patients (and potentially also their carers) and which relate to "how the patient feels, functions or survives". In the context of advanced/ metastatic cancer, the key matter of concern is often whether the use of a given health technology leads to improvements in overall survival (OS; a final endpoint) compared to existing standard treatments. However, the estimation of treatment effects on OS may be subject to numerous problems, including potential confounding resulting from the use of post-progression treatments, insufficient study follow-up resulting in data immaturity or simply that data on OS have not been collected. In such instances, determining the impact of health technologies becomes more challenging and may rely on the use of surrogate endpoints to substitute for, and predict, a final patient-relevant clinical outcome. ${ }^{2}$ Potentially relevant surrogate endpoints vary according to tumour type and site, but commonly include progression-free survival (PFS), time to progression (TTP), and response-based outcomes, which may include overall response rate (ORR), different levels of response (e.g. complete response [CR], partial response [PR] or very good partial response [VGPR]) and duration of response (DoR). These surrogate endpoints are often considered attractive as they typically require smaller sample sizes, occur faster and are less expensive to collect in clinical trials compared with final outcomes, thereby reducing costs associated with data collection and expediting the time required for bringing new technologies to market.

It has been recognised in the literature that the reliance on surrogates may lead to invalid conclusions regarding the net health effects of technologies, which in turn have the potential to lead to patient harm. ${ }^{3}$ Much of the published literature around the use of surrogate endpoints has focussed on the development and application of frameworks for their validation. ${ }^{4,5}$ In his seminal paper, Prentice ${ }^{4}$ put forward stringent criteria for the validation of surrogate endpoints in phase 3 trials. In general terms, these criteria require that the surrogate endpoint must be a correlate of the net effect of treatment on the final clinical outcome-in other words, there must be a single pathway from the treatment to the true endpoint, which is mediated exclusively by the surrogate endpoint. ${ }^{6}$ Applied surrogate validation studies commonly adopt a meta-analytic (meta-regression) approach based on multiple studies in order to assess whether the apparent relationship

${ }^{1}$ ScHARR, University of Sheffield, Sheffield, UK

Correspondence: Katy Cooper (k.l.cooper@sheffield.ac.uk)

Received: 26 February 2020 Revised: 29 July 2020 Accepted: 18 August 2020

Published online: 11 September 2020 
between the surrogate and the final endpoint remains constant in the presence of various sources of heterogeneity, such as differences in patient population, study design and treatments received. ${ }^{5}$

Based on the NIH Biomarkers Definition Working Group's preferred terms and definitions ${ }^{7}$ and the 2001 Journal of the American Medical Association (JAMA) User's Guide, ${ }^{8}$ Taylor and Elston ${ }^{9}$ proposed a hierarchy of levels of surrogate validation. Level 3 of the hierarchy relates to biological plausibility-this is the weakest form of validation and is typically based on pathophysiological studies and/or an understanding of the disease process. Level 2 requires the presence of a consistent association between the surrogate outcome and the final endpoint; this may be assessed using observational studies or arm-based analyses of trials, which have measured both the surrogate and the final outcome. This level of validation requires an assessment of the individual-level (absolute) association between endpoints and is usually undertaken using correlation analysis. Level 1 of the hierarchy represents the strongest level of surrogate validation: in order to achieve this level of validation, the treatment effect on the surrogate must correspond to the treatment effect on the final outcome. Demonstrating this level of validity requires an analysis of correlation in terms of treatment effects between arms based on data from RCTs (trial-level association). Other validation frameworks have been proposed to assess the strength of association between surrogate and final endpoints. These include the criteria proposed by the German Institute of Quality and Efficiency in Health Care ${ }^{10}$ (IQWiG; based on the treatment effect association only) and the BiomarkerSurrogate Evaluation Schema criteria ${ }^{11}$ (BSES2; based on both absolute and treatment effect associations). These frameworks differ in terms of the types of analyses and the strength of the relationship required to determine the reliability of the surrogate.

This systematic review summarises published meta-regression studies reporting correlation and regression analyses for the strength of the association between response-based outcomes and PFS, TTP or OS in (primarily) advanced or metastatic cancer, across any tumour site, in order to assess whether response-based outcomes may be considered as valid surrogates for PFS, TTP or OS.

\section{METHODS}

Inclusion and exclusion criteria

Inclusion was restricted to articles reporting meta-analyses or meta-regressions across multiple studies and reporting the strength of association between response outcomes (ORR, CR, PR, VGPR or DoR) and either PFS, TTP or OS. The included metaregressions could themselves include RCTs and/or single-arm studies. However, individual reports analysing single trials or single cohorts were excluded from this review. Included metaanalyses could report absolute associations and/or treatment effect associations. These associations had to be reported as a correlation coefficient (e.g. Pearson's $r$ or Spearman's $r_{s}$ ) and/or a coefficient of determination $\left(R^{2}\right)$ between relevant outcomes.

Studies of any cancer and any treatment were included. The review focussed mainly on studies of advanced or metastatic cancers (and/or treatment with palliative intent), as these studies were more likely to report PFS and OS. However, studies reporting relevant outcomes were included even where the stage was not specifically restricted to advanced/metastatic disease for all patients or where this was unclear (this applied particularly to haematological cancers). Studies were excluded if they explicitly referred to adjuvant or neo-adjuvant treatment, or treatments that are given with curative intent. Studies were only included if they were written on English or contained sufficient detail in English.

The review protocol is registered on PROSPERO with registration number CRD42019127606.
Search strategy

Five databases (MEDLINE, EMBASE, Web of Science, the Cochrane Database of Systematic Reviews and CINAHL) were searched from inception to March 2019. Search terms included cancer terms AND response terms AND terms for PFS, TTP and/or OS AND terms for regression, correlation, prediction, association or relationship AND terms for endpoint and/or surrogate. Search results were limited to the English language and to studies undertaken in humans. The MEDLINE search strategy is provided in Supplementary Information 1. In addition, a citation search was undertaken based on two existing meta-reviews of surrogate relationships; this identified studies that have cited any of the 48 articles included in the review by Fischer et al. ${ }^{12}$ and/or any of the 19 articles included in the review by Davis et al. ${ }^{13}$ In addition, relevant existing meta-reviews, including Fischer et al., ${ }^{12}$ Davis et al., ${ }^{13}$ Savina et al., ${ }^{14}$ Haslam et al. ${ }^{15}$ and any reviews identified during searching, were checked for relevant studies.

Scoring the strength of association: IQWiG and BSES2 scoring In this review, two sets of published criteria were used to assess the strength of association between surrogate and final endpoints: the IQWiG criteria $^{10}$ and the BSES2 criteria. ${ }^{11}$

The IQWiG criteria ${ }^{10}$ are based on the correlation coefficient $(r)$ for the treatment effect association. Where $r$ was not reported, it was calculated as the square root of $R^{2}$, if available. As the medium score bracket was not clearly defined, slight modifications were made to the IQWiG criteria based on the approach used in the previous review by Savina et al. ${ }^{14}$ (Supplementary Table 1). The IQWiG score was generated based on the magnitude of $r$, irrespective of its sign (i.e. a negative correlation could generate a high score). The IQWiG criteria were scored as follows: high (lower confidence interval of $r$ is $\geq 0.85)$; medium $+(r \geq 0.85$ with no reported confidence interval or $r \geq 0.85$ with wide confidence intervals [lower limit $<0.85$ ]); medium (0.85 $>r \geq 0.7$ and upper confidence interval of $r$ is $\geq 0.7$ and lower confidence interval of $r$ is $<0.85$, or $0.85>r \geq 0.7$ with no reported confidence interval); or low (upper confidence interval of $r$ is $<0.7$ or $r<0.7$ with no reported confidence interval).

The BSES2 criteria ${ }^{11}$ require $R^{2}$ values for both the absolute and treatment effect associations. Where $R^{2}$ was not reported, it was calculated as the square of $r$, if available. BSES2 criteria were used as an adaptation from the original BSES criteria, as described in Savina et al. ${ }^{14}$ The original BSES criteria require $R^{2}$ for both individual and treatment effect associations and a value for the surrogate threshold effect (STE). Since so few articles report STE, this review used BSES2, which does not require the STE. The BSES2 criteria were scored as follows: excellent $\left(R^{2}\right.$ [treatment effect] $\geq 0.6$ and $R^{2}$ [absolute] $\left.\geq 0.6\right) ;$ good $\left(R^{2}\right.$ [treatment effect] $\geq 0.4$ and $R^{2}$ [absolute] $\geq 0.4)$; fair $\left(R^{2}\right.$ [treatment effect] $\geq 0.2$ and $R^{2}$ [absolute] $\geq 0.2)$; poor $\left(R^{2}\right.$ [treatment effect] $<0.2$ and/or $R^{2}$ [absolute] $<0.2$ ). Further details on the IQWiG and BSES2 scoring systems are provided in Supplementary Tables 1 and 2.

\section{Study selection and data extraction}

Titles and abstracts of articles retrieved by the search were examined by one reviewer and a subset was checked by a second reviewer early in the process, followed by a discussion to ensure consistency in the selection decisions. Full texts were examined by one reviewer and a subset was checked by a second reviewer, with any discrepancies resolved through discussion.

Data were extracted by one reviewer and all data were checked by a second reviewer. Data were extracted relating to study design, participant characteristics, surrogate and final endpoints analysed, methods for correlation and regression, and results including absolute associations, associations between treatment effects, STE and regression equations.

\section{Data synthesis}

Data were presented in a narrative synthesis. Plots were constructed to illustrate the reported associations within each study. Some of the 


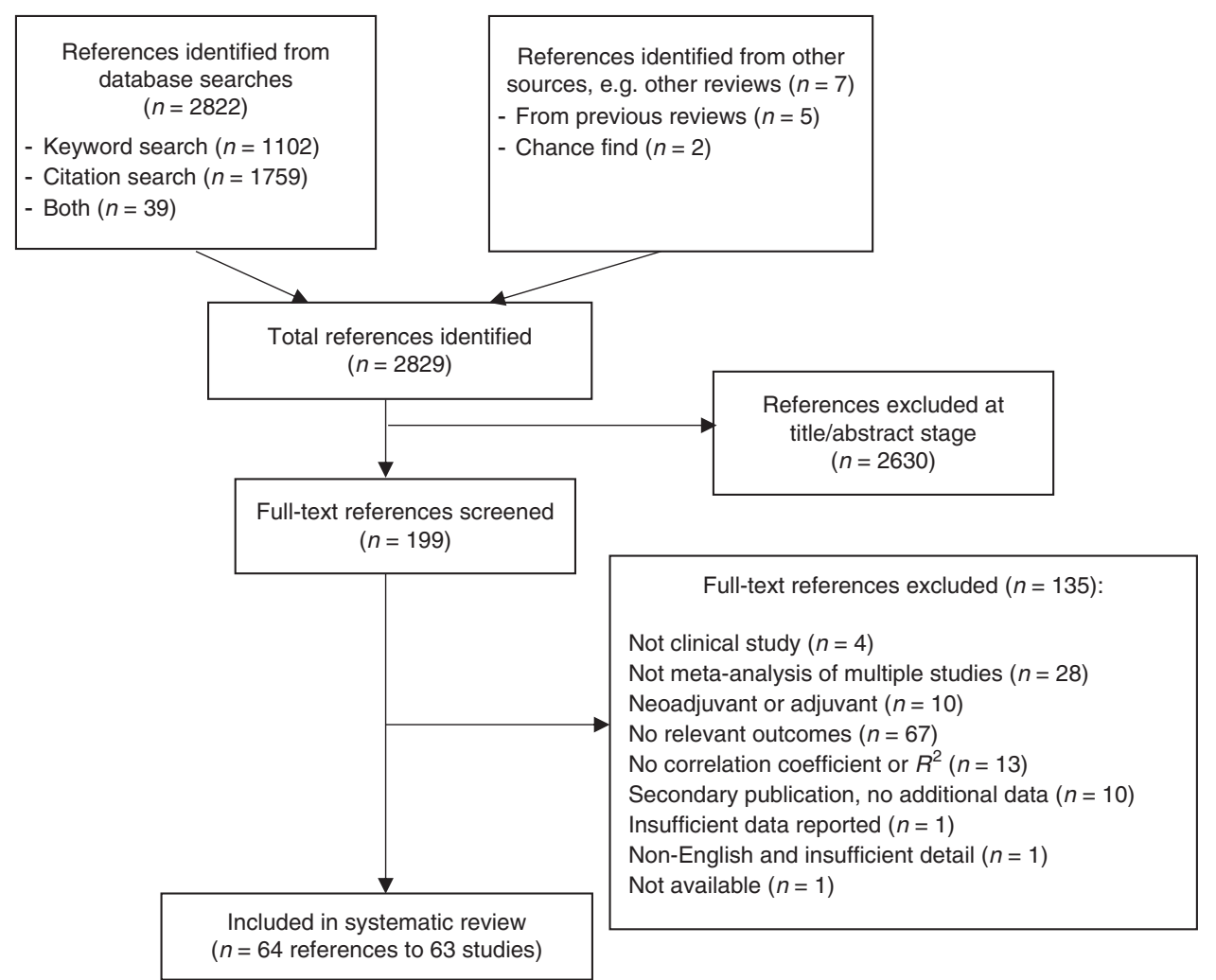

Fig. 1 PRISMA flow diagram for study inclusion. Illustrates the number of references retrieved from the literature searches and included/ excluded at each stage of screening.

included meta-regression studies reported multiple subgroup analyses with differing results. Therefore, each horizontal row in the plots illustrates the range of reported associations across all subgroup analyses within a single meta-regression study. Where an included meta-regression study reported on more than one cancer type, these are shown on separate rows on the plots.

For associations between absolute values of endpoints, the plots show the range of correlation coefficients per study, across all subgroup analyses. All types of correlation coefficient were included, for example, Pearson's $r$ and Spearman's $r_{s}$. If no correlation coefficient was reported, then Pearson's $r$ was calculated as the square root of $R^{2}$, if available.

For associations between treatment effects, the plots show the range of regression coefficients of determination $\left(R^{2}\right)$ per study, across all subgroup analyses. The plots include both adjusted and unadjusted $R^{2}$ values, as well as values from weighted and unweighted regressions. For studies in which $R^{2}$ was not reported, this was calculated as the square of the Pearson's $r$ correlation coefficient, if available. $R^{2}$ was not calculated from other correlation coefficients such as Spearman, or where the method of correlation was unclear.

Quality assessment

Included meta-regression studies were assessed for methodological quality based on key criteria from the AMSTAR- $2^{16}$ and ReSEEM $^{17}$ checklists most relevant to our review.

\section{RESULTS}

Number of included meta-regression studies

The literature search generated 2829 citations (Fig. 1), of which 2630 were excluded during the review of titles and abstracts and a further 135 excluded during the review of full texts. In total, 63 studies (within 64 references) were included in the review. ${ }^{18-81}$
Characteristics of included meta-regression studies

Summaries of study characteristics and reported data types are provided in Supplementary Tables 3 and 4, respectively, while full details of study characteristics for each of the 63 included studies are provided in Supplementary Table 5.

The most commonly reported surrogate relationships were ORR to OS (57 studies), ORR to PFS (22 studies), CR to OS (8 studies) and CR to PFS (7 studies). Other response outcomes (DoR, PR and VGPR/CR) were only reported in one to two studies each. Twenty different cancer types were analysed, the most common being NSCLC (16 studies), CRC (10 studies), various solid tumours ( 8 studies) and breast cancer (5 studies). Disease stage was advanced/metastatic in 43 studies and unclear in 9 studies, while the remainder (11 studies) gave other descriptions mostly indicating advanced, extensive or recurrent disease. Treatment was first line in 23 studies, later lines or combinations of lines in 32 studies and not reported in 8 studies. Treatment type was chemotherapy in 21 studies, immune checkpoint inhibitors in 9 studies, targeted therapy in 8 studies and various other treatment combinations in the remainder.

The various meta-regressions included between 4 and 191 primary studies and between 407 and 44,125 patients each. The majority of meta-regressions $(N=44)$ included only RCTs, while 17 included both RCTs and single-arm studies and 2 included only single-arm studies. Most meta-regressions $(N=58)$ analysed aggregate data (e.g. medians or other summary measures per study arm), while 5 analysed individual patient data (IPD). Across all meta-regressions, 32 reported absolute (individual-level) associations, 38 reported treatment effect (trial-level) associations and only 4 reported the STE.

Methodological quality of included meta-regression studies Methodological quality of the included studies is shown in Supplementary Table 6. All studies had clear inclusion criteria; 


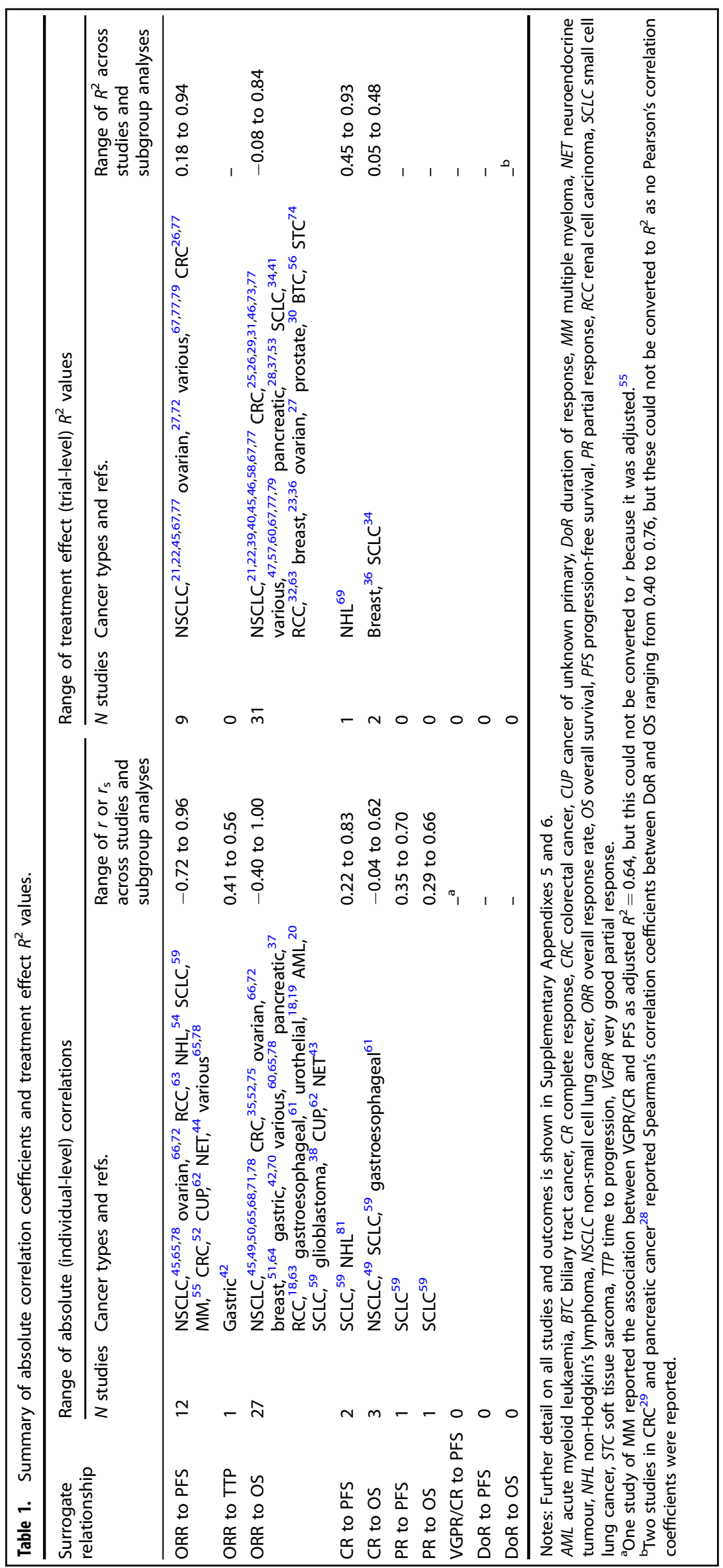




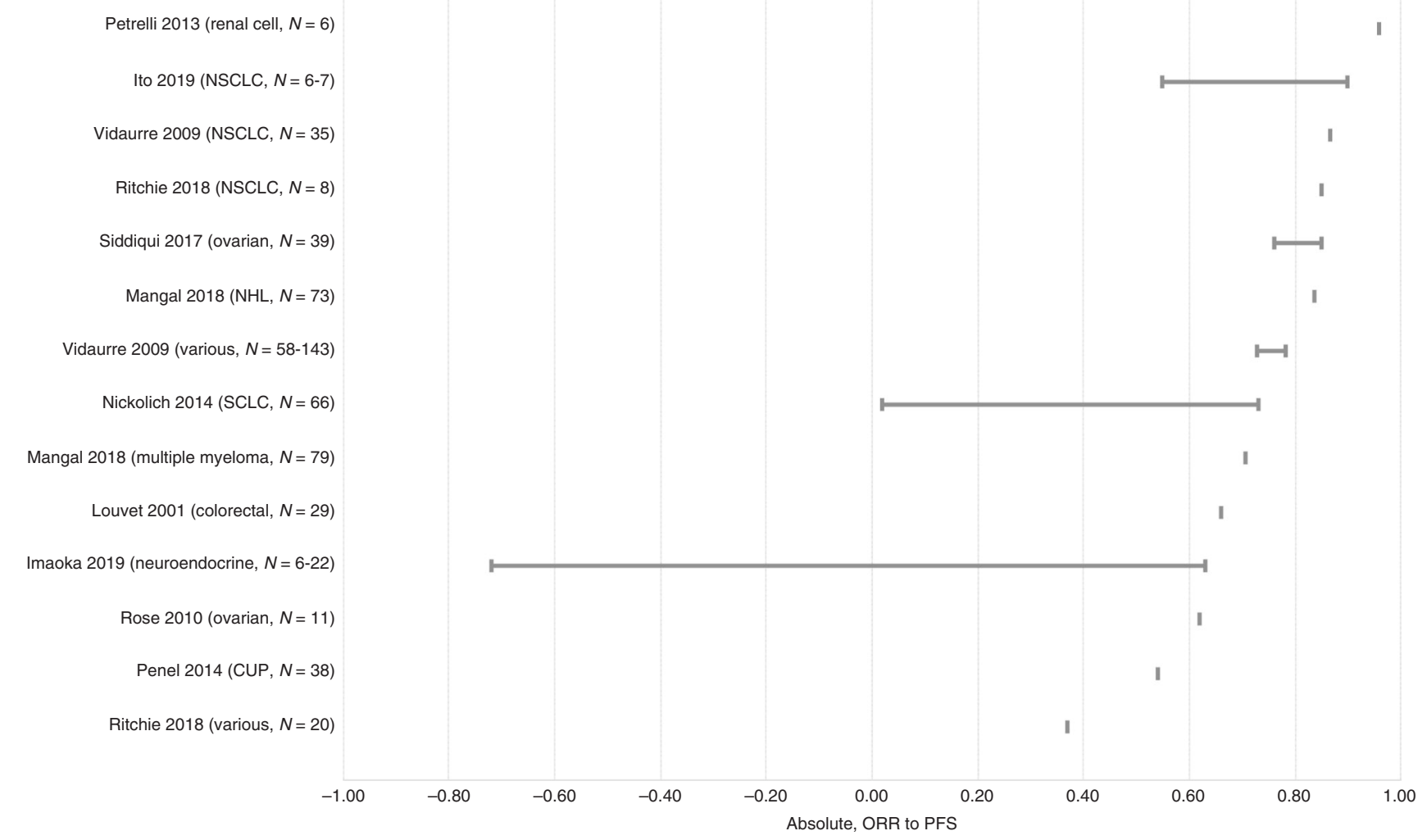

Fig. 2 Correlation ( $r$ or $\boldsymbol{r}_{\mathrm{s}}$ ) between absolute (individual-level) values of ORR and PFS. For each study, the plot illustrates the range of correlation coefficients across all subgroup analyses. $N$ represents the number of studies included in each meta-regression. CUP cancer of unknown primary, NHL non-Hodgkin's lymphoma, NSCLC non-small cell lung cancer, ORR overall response rate, PFS progression-free survival, SCLC small cell lung cancer.

$65 \%$ reported a comprehensive literature search; and $98 \%$ reported a correlation coefficient or $R^{2}$ value (the one study not reporting these was included as it reported a regression slope). However, only $27 \%$ reported duplicate study selection; $48 \%$ reported duplicate data extraction or checking; and $13 \%$ reported a risk of bias assessment of included studies. In addition, only $37 \%$ explored heterogeneity through subgroup analyses, and only $40 \%$ reported confidence intervals around the correlation coefficient or $R^{2}$.

Results of included studies

The reported associations between surrogate and final endpoints are summarised in Table 1 and illustrated in Figs. 2-5. Full results for each included meta-regression study are provided in Supplementary Table 7 (for absolute associations) and Supplementary Table 8 (for treatment effect associations).

Absolute (individual-level) correlation and regression

The range of absolute (individual-level) correlation coefficients is summarised in Table 1 and illustrated in Fig. 2 (for the association between ORR and PFS) and Fig. 3 (for the association between ORR and OS). Some of the included meta-regression studies reported multiple subgroup analyses with differing results. Therefore, each horizontal row in the plots illustrates the range of correlation coefficients across all subgroup analyses within a single meta-regression study. Where an included meta-regression reported on more than one cancer type, these are shown on separate rows on the plots. It is worth noting that the included meta-regression studies differed in terms of various factors, such as the number of included primary studies (shown as $N$ on the plots), treatment type, line of treatment and precise clinical population (full details in Supplementary Table 7).
ORR and PFS (or TTP). The reported correlation coefficients (Pearson's $r$ or Spearman's $r_{s}$ ) between absolute ORR and PFS ranged from -0.72 to 0.96 , based on multiple analyses within 12 studies across 10 cancer types ${ }^{44,45,52,54,55,59,62,63,65,66,72,78}$ (Fig. 2 and Table 1). Across those studies that report only a single analysis, the correlation coefficient was generally $>0.60$; however, some estimates were lower. Confidence intervals around the correlation coefficients were rarely reported. Few separate meta-regressions reported on the same tumour site, hence it is difficult to assess whether ORR may be a more reliable surrogate in certain cancer types than others. One study reported on ORR and TTP (gastric cancer; correlation $r_{\mathrm{s}}=$ $0.41-0.56$ across subgroup analyses, not shown on the plot). ${ }^{42}$

ORR and OS. The reported correlation coefficients between absolute ORR and OS ranged from -0.40 to 1.00 , based on 27 studies across 15 cancer types ${ }^{18-20,35,37,38,42,43,45,49-52,59-66,68,70-72,75,78}$ (Fig. 3 and Table 1). Confidence intervals around the correlation coefficients, where reported, were generally fairly wide. The majority of correlation coefficients were $>0.40$; however, several estimates were lower. Neither the correlation coefficients reported from multiple analyses within the same study, nor those reported across separate studies, suggested a clear pattern by cancer type.

$C R$ and PFS or OS. The correlation coefficients between absolute $\mathrm{CR}$ and PFS in two studies of small cell lung cancer (SCLC) $)^{59}$ and non-Hodgkin's lymphoma (NHL) ${ }^{81}$ ranged from 0.22 to 0.83 , while the correlation coefficients between absolute $C R$ and $O S$ ranged from -0.04 to 0.62 , based on three studies of NSCLC, ${ }^{49} \mathrm{SCLC}^{59}$ and

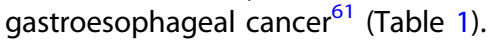

$P R$ and PFS or OS. The correlation coefficient between absolute $\mathrm{PR}$ and PFS ranged from 0.35 to 0.70 across subgroup analyses 


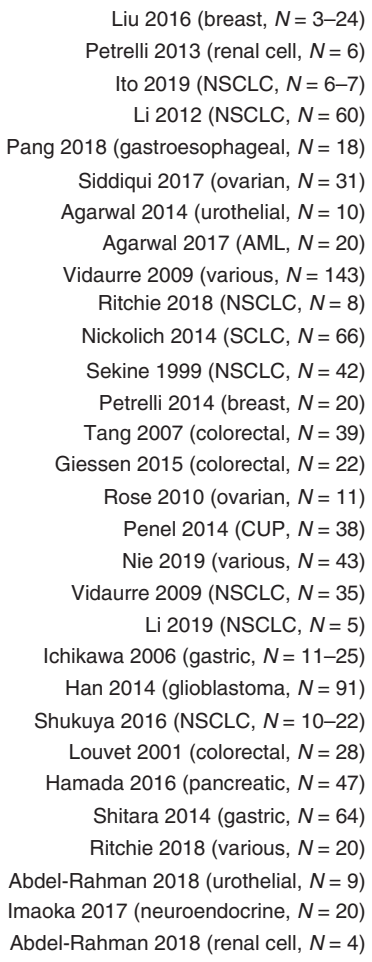

Liu 2016 (breast, $N=3-24$ )

Petrelli 2013 (renal cell, $N=6$ )

Ito 2019 (NSCLC, $N=6-7$ )

Li 2012 (NSCLC, $N=60$ )

Pang 2018 (gastroesophageal, $N=18$ )

Siddiqui 2017 (ovarian, $N=31$ )

Agarwal 2014 (urothelial, $N=10$ )

Agarwal 2017 (AML, $N=20$ )

Vidaurre 2009 (various, $N=143$ )

Ritchie 2018 (NSCLC, $N=8$ )

Nickolich 2014 (SCLC, $N=66$ )

Sekine 1999 (NSCLC, $N=42$ )

Petrelli 2014 (breast, $N=20$ )

Tang 2007 (colorectal, $N=39$ )

Giessen 2015 (colorectal, $N=22$ )

Rose 2010 (ovarian, $N=11$ )

Penel 2014 (CUP, $N=38$ )

Nie 2019 (various, $N=43$ )

Vidaurre 2009 (NSCLC, $N=35$ )

Li 2019 (NSCLC, $N=5$ )

Ichikawa 2006 (gastric, $N=11-25$ )

Han 2014 (glioblastoma, $N=91$ )

Shukuya 2016 (NSCLC, $N=10-22$ )

Louvet 2001 (colorectal, $N=28$ )

Hamada 2016 (pancreatic, $N=47$ )

Shitara 2014 (gastric, $N=64$ )

Ritchie 2018 (various, $N=20$ )

Abdel-Rahman 2018 (urothelial, $N=9$ )

Imaoka 2017 (neuroendocrine, $N=20$ )

Abdel-Rahman 2018 (renal cell, $N=4$ )
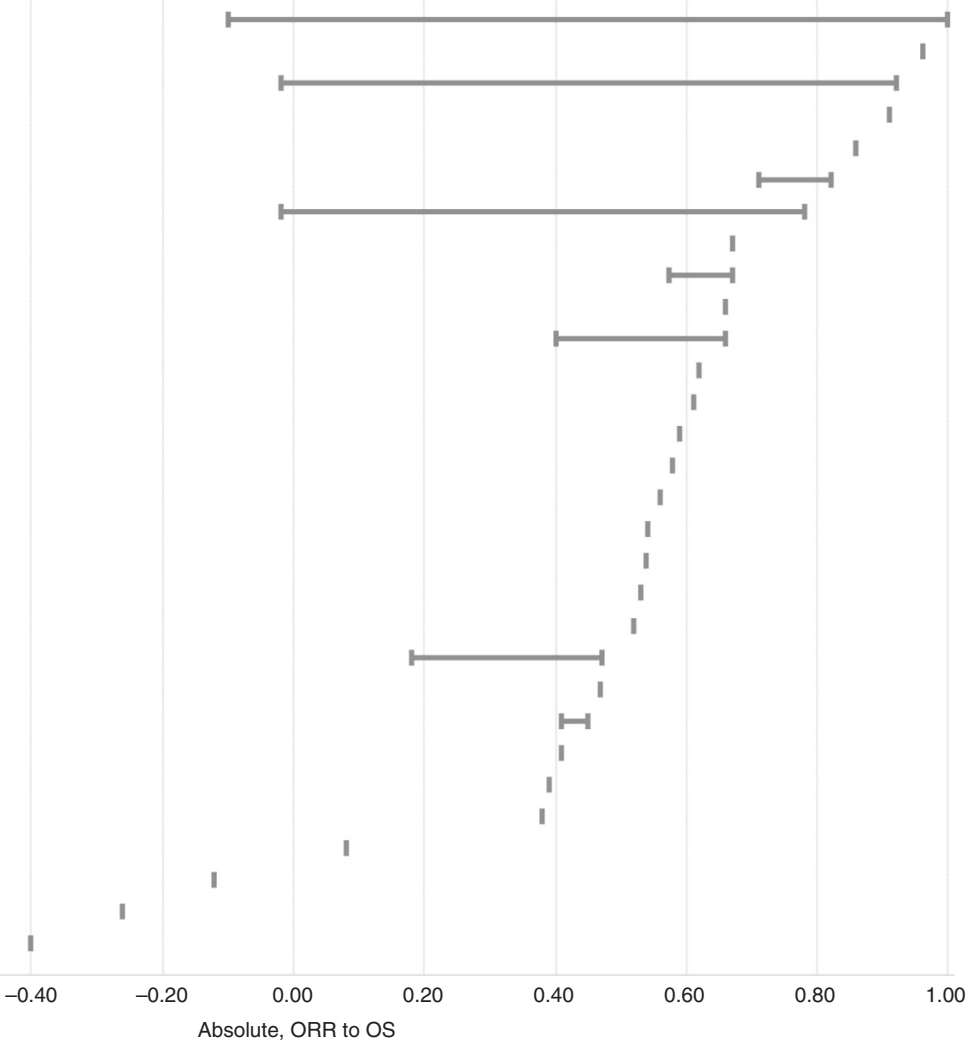

Fig. 3 Correlation ( $r$ or $\boldsymbol{r}_{\mathbf{s}}$ ) between absolute (individual-level) values of ORR and OS. For each study, the plot illustrates the range of correlation coefficients across all subgroup analyses. $N$ represents the number of studies included in each meta-regression. AML, acute myeloid leukaemia, CUP cancer of unknown primary, NSCLC non-small cell lung cancer, ORR overall response rate, OS overall survival, SCLC small cell lung cancer.

within one study of $\mathrm{SCLC}^{59}$ while the highest correlation coefficient between absolute PR and OS ranged from 0.29 to 0.66 in the same study ${ }^{59}$ (Table 1 ).

DoR and PFS or OS. No studies reported on the absolute association between DoR and PFS or OS.

Treatment effect (trial-level) correlation and regression

The range of treatment effect (trial-level) $R^{2}$ values is summarised in Table 1 and illustrated in Fig. 4 (for the association between ORR and PFS) and Fig. 5 (for the association between ORR and OS). Each horizontal row in the plots illustrates the range of $R^{2}$ values across all subgroup analyses within a single meta-regression study. Where an included meta-regression reported on more than one cancer type, these are shown separately on the plots. It is worth noting that the meta-regressions differed in terms of the number of included primary studies (shown as $N$ on the plots), treatment type, line of treatment and precise clinical population (full details in Supplementary Table 8).

ORR and PFS. The regression $R^{2}$ values for the treatment effect association between ORR and PFS ranged from 0.18 to 0.94 , based on nine studies across five cancer types: NSCLC, ${ }^{21,22,45,67,77}$ ovarian cancer, ${ }^{27,72}$ colorectal cancer ${ }^{26,77}$ and various solid tumours ${ }^{67,77,79}$ (Fig. 4 and Table 1). The majority of $R^{2}$ values were above 0.40 . The $R^{2}$ values reported from multiple analyses within the same study, and those reported across separate studies, did not suggest a clear pattern by cancer type. Confidence intervals around the $R^{2}$ values, where reported, were generally fairly wide.

ORR and OS. The regression $R^{2}$ values for the treatment effect association between ORR and OS ranged from
-0.08 to 0.84 , based on 31 studies across 11 cancer types $^{21-23,25-32,34,36,37,39-41,45-47,53,56-58,60,63,67,73,74,77,79}$ (Fig. 5 and Table 1). With the exception of one analysis, ${ }^{77}$ all $R^{2}$ values were below 0.60 . The $R^{2}$ values reported from multiple analyses within the same study, and those reported across separate studies, did not suggest a clear pattern by cancer type. Confidence intervals around the $R^{2}$ values, where reported, were generally wide.

$C R$ and PFS or OS. The regression $R^{2}$ for the treatment effect association between CR and PFS ranged from 0.45 to 0.93 across subgroup analyses within one study of $\mathrm{NHL}^{69}$ while the regression $R^{2}$ for the treatment effect association between CR and OS within two studies of breast cancer ${ }^{36}$ and $\mathrm{SCLC}^{34}$ ranged from 0.05 to 0.48 (Table 1).

$P R$ and PFS or OS. No studies reported the treatment effect association between PR and PFS or OS.

DoR and PFS or OS. No studies reported $R^{2}$ between DoR and OS or PFS. Two studies in colorectal cancer ${ }^{29}$ and pancreatic cancer ${ }^{28}$ reported Spearman's correlation coefficients between DoR and OS ranging from 0.40 to 0.76 (Table 1 ).

Influence of clinical and study factors on association The impact of the following patient and study factors on the association between ORR and OS was explored: treatment line; treatment type; response criteria; adjustment of OS for crossover and post-progression treatments; and aggregate versus IPD data (Supplementary Table 9). No clear effect on the association between ORR and OS was identified for any individual factor. However, this analysis was limited by the small number of publications assessing each factor within 
Blumenthal 2015 (NSCLC, $N=11-14)$

Ito 2019 (NSCLC, $N=6-7$ )

Blumenthal 2017 (NSCLC, $N=25)$

Roviello 2017 (various, $N=17$ )

Tsujino 2010 (colorectal, $N=7$ )

Ciani 2015, Elia 2018 (colorectal, $N=7-33$ )

Colloca and Venturino 2017 (ovarian, $N=15-29$ )

Tsujino 2010 (various, $N=17$ )

Wilkerson + Fojo 2009 (various, $N=66$ )

Roviello 2017 (NSCLC, $N=7$ )

Siddiqui 2017 (ovarian, $N=39$ )

0.00

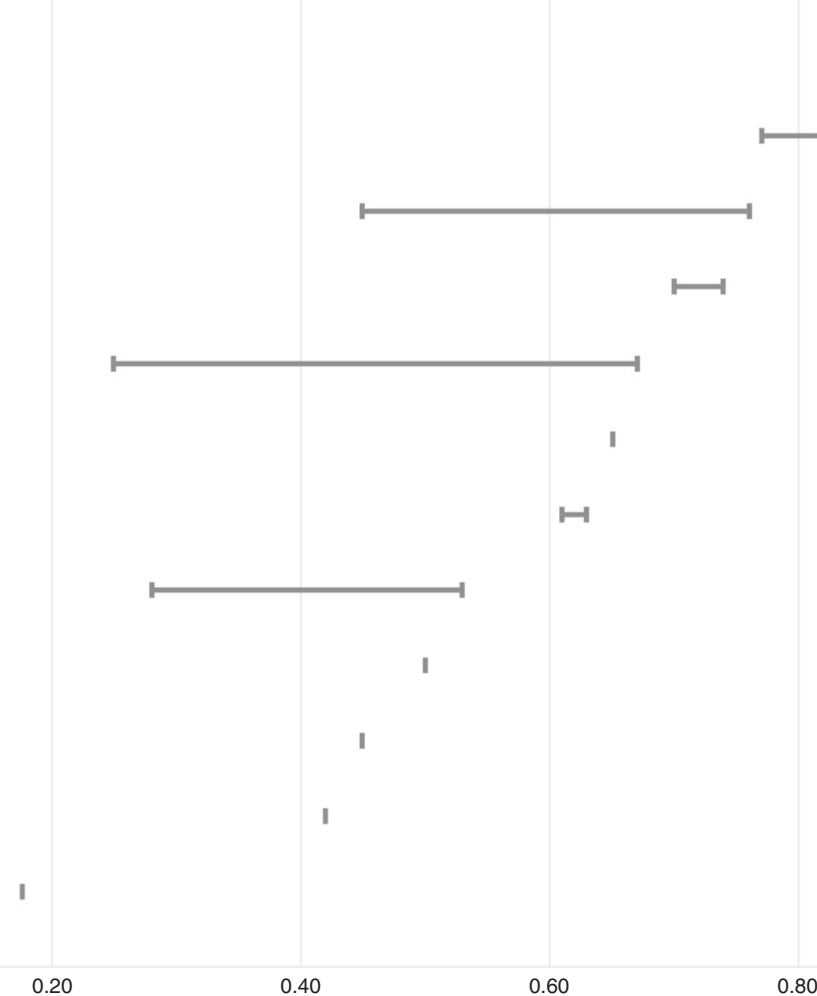

Treatment effect $R^{2}$, ORR to PFS
0.80

1.00

Fig. 4 Regression $\boldsymbol{R}^{2}$ between treatment effects (trial-level) for ORR and PFS. For each study, the plot illustrates the range of correlation coefficients across all subgroup analyses. $N$ represents the number of studies included in each meta-regression. NSCLC non-small cell lung cancer, ORR overall response rate, PFS progression-free survival.

each cancer, and the wide ranges of associations observed for each.

Five of the 63 included meta-analyses analysed IPD rather than aggregate data; two in breast cancer ${ }^{23,24}$, one in colorectal cancer ${ }^{25}$, one in $\mathrm{NHL}^{69}$ and one in ovarian cancer ${ }^{66}$. The associations reported in these studies were not noticeably different to those in other studies (see Figs. 2-5).

\section{Regression equations}

Regression equations were reported in 14 studies for the relationship between ORR and OS; of these, four reported absolute associations ${ }^{42,52,72,76}$ and ten reported treatment effect associations. ${ }^{31-33,36,41,46,56,58,67,77}$ Regression equations were also reported in eight studies for the relationship between ORR and PFS; of these, four reported absolute associations ${ }^{52,54,72,76}$ and four reported treatment effect associations. ${ }^{24,33,67,77}$ These analyses spanned 10 cancer types. Full details are provided in Supplementary Tables 10 and 11 . There was substantial variation in the effect measures used for both the surrogate and final outcomes; for example, difference in medians, hazard ratio (HR), odds ratio (OR), log-transformed or not. None of the included studies attempted to externally validate their regression equations for the relationship between response and other outcomes.

\section{Surrogate threshold effect}

The STE-the smallest treatment effect on the surrogate that predicts a non-zero treatment effect on the true endpoint ${ }^{82}$ - was reported in only four studies (Supplementary Table 12). ${ }^{26,39,69,77}$ For the relationship between ORR and PFS, one study ${ }^{77}$ in various solid tumours reported that a difference in ORR of $15 \%$ would be required to predict a non-zero treatment effect on the HR for PFS. For the relationship between ORR and OS, two studies in various solid tumours ${ }^{77}$ and $\mathrm{NSCLC}^{39}$ reported that a difference in ORR of
$21 \%$ and $55 \%$, respectively, would be required to predict a nonzero treatment effect on the HR for OS, while one study ${ }^{39}$ also reported that a difference in ORR of $41 \%$ would be required to predict a non-zero treatment effect on the difference in median OS. A further study in colorectal cancer ${ }^{26}$ reported that an OR for ORR of 0.28 would be required to predict a non-zero treatment effect on the OR for OS. Finally, for the relationship between $\mathrm{CR}$ and PFS, one study in $\mathrm{NHL}^{69}$ reported that an $\mathrm{OR}$ for $\mathrm{CR}$ (at 30 months) of 1.56 would be required to predict a non-zero treatment effect on the HR for PFS.

IQWiG and BSES2 scores for the strength of association IQWiG and BSES2 scores for the strength of association between surrogate and final endpoints were calculated for all reported subgroup analyses with sufficient data; therefore, meta-regression studies that reported more subgroups are more strongly represented in this analysis. These data are presented graphically in Supplementary Figs. 1 and 2.

In terms of IQWiG scores, of 202 analyses (across 63 studies), 0 (0\%) scored high, $15(7 \%)$ scored medium+, 26 (13\%) scored medium, 76 (38\%) scored low and 85 (42\%) were not evaluable. In terms of BSES2 scores, of 202 analyses (across 63 studies), 0 (0\%) scored excellent, 3 (1\%) scored good, 3 (1\%) scored fair, 7 (3\%) scored poor and 189 (94\%) were not evaluable.

\section{DISCUSSION}

This systematic review summarises published meta-regression studies reporting correlation and regression analyses for the strength of the association between response outcomes and PFS, TTP or OS across different types of cancer. In total, the review included 63 studies across 20 cancer types. The most commonly analysed relationships were between ORR and either PFS or OS. 


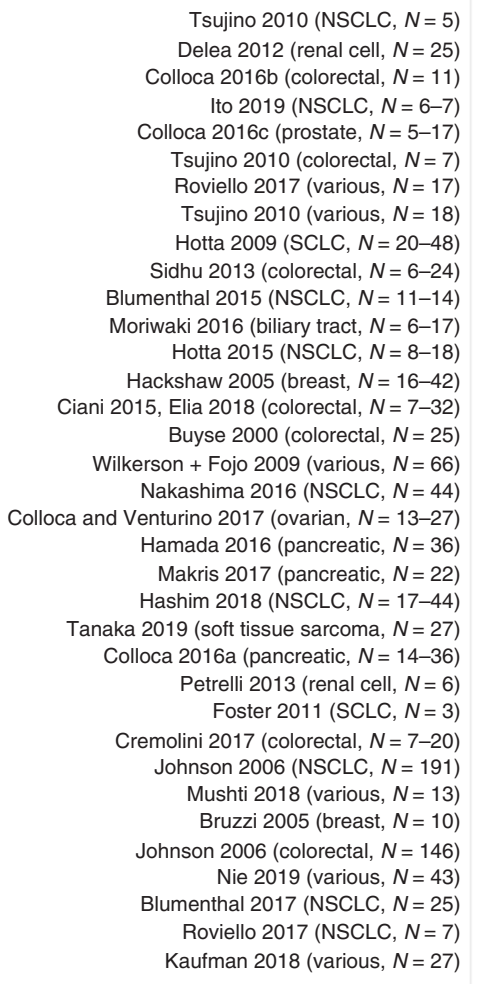

$-0.20$

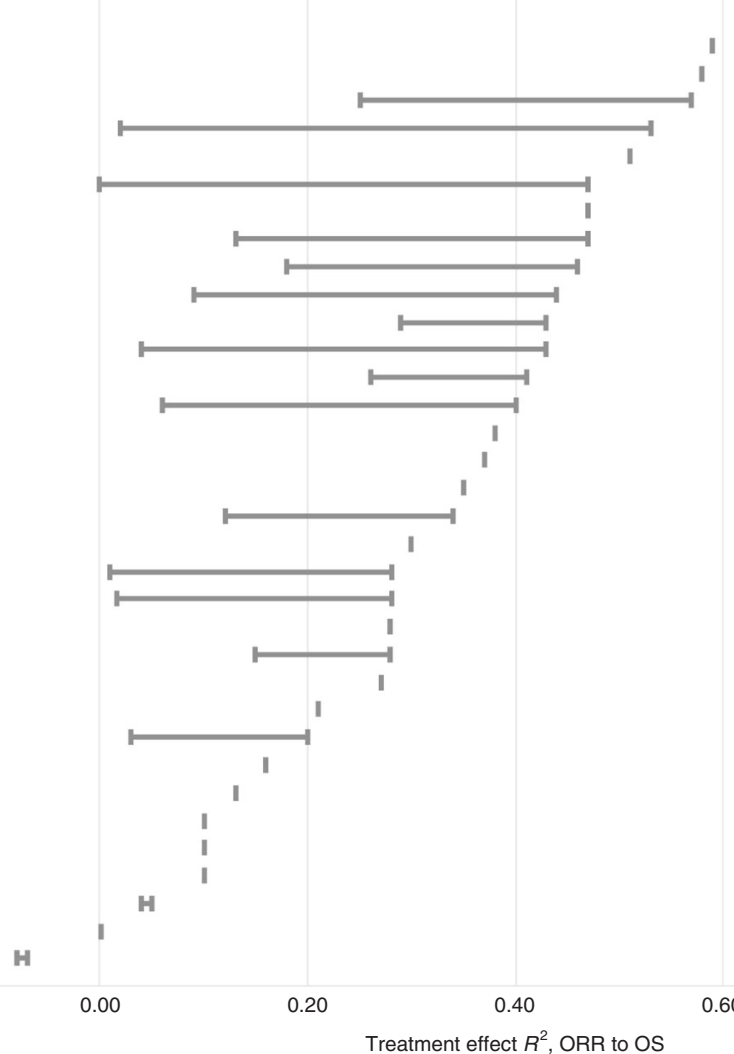

Fig. 5 Regression $R^{2}$ between treatment effects (trial-level) for ORR and OS. For each study, the plot illustrates the range of correlation coefficients across all subgroup analyses. $N$ represents the number of studies included in each meta-regression. NSCLC non-small cell lung cancer, ORR overall response rate, OS overall survival, SCLC small cell lung cancer.

For the association between ORR and PFS, the majority of reported correlation coefficients between absolute values were $>0.60$ (range -0.72 to 0.96 ). For association between treatment effects on ORR and PFS, the majority of regression $R^{2}$ values were $>0.40$ (range 0.18-0.94). The association between ORR and OS appeared weaker than that between ORR and PFS; while the majority of reported correlation coefficients between absolute values were $>0.40$, several estimates were lower (range -0.40 to 1.00). For association between treatment effects on ORR and OS, all regression $R^{2}$ values except one were below 0.60 (range -0.08 to 0.84 ).

There was no clear pattern by cancer type for either the absolute or treatment effect associations, based on both multiple analyses within the same study and results across separate studies. Confidence intervals around the reported correlation coefficients and $R^{2}$ values were generally wide and often not reported.

Strength of association across all subgroup analyses within all included meta-regression studies was assessed using the IQWiG and BSES2 scoring systems. The majority of analyses were not evaluable due to the lack of required data. Of those analyses that could be scored, scores were relatively low, suggesting that response-based endpoints may be poor surrogates for OS.

Previous systematic reviews of surrogate endpoints in advanced cancer have been published. Savina et al. ${ }^{14}$ and Haslam et al. ${ }^{15}$ have reported systematic reviews of meta-analyses assessing any endpoint as a surrogate for OS. Both these reviews also assessed the strength of association using surrogate validation frameworks; both studies used adaptations of the IQwiG framework, and Savina et al. ${ }^{14}$ also used the BSES2 framework. These previous reviews generally focussed on the main analyses presented within individual meta-analyses (usually that with the largest number of patients). Similar to our review, these previous reviews suggested that response-based outcomes are likely to be poor surrogates for OS. Our systematic review focusses exclusively on response-based surrogates; it includes a comprehensive search to identify relevant studies, considers PFS as a potential final endpoint as well as OS, is more up to date, includes a greater number of studies and reports results for the full breadth of analyses reported in the included meta-regression studies compared with these previous reviews. This provides a more complete picture of the extent of heterogeneity in reported relationships across the full range of meta-analyses across each cancer area. This additional breadth provides a better basis to inform judgements about the validity of response-based endpoints as a surrogate for PFS or OS.

The review is subject to a number of limitations. The reported data were highly heterogeneous in terms of effect measure and method of analysis. Therefore, some simplifying assumptions had to be made to allow the data to be summarised. For example, correlation coefficients were summarised regardless of method (Pearson's, Spearman's or other); $R^{2}$ values were summarised irrespective of whether or not the regression was weighted and whether or not the $R^{2}$ was adjusted; and for treatment effect associations, $R^{2}$ values were summarised regardless of the effect measure (e.g. HR, OR, difference in medians). In addition, only five studies used IPD rather than aggregate data in their analysis; this is a limitation of the analyses conducted in the majority of metareviews. A recent review by Xie et al. ${ }^{17}$ highlighted wide variability in reporting standards across surrogate evaluation metaregression studies; future analyses should attempt to adhere to current best practice, for example, the reporting of surrogate endpoint evaluation using meta-analyses (ReSEEM) guidelines in order to improve the quality of these analyses. ${ }^{17}$

It should further be noted that while meta-regression has been widely used for the purpose of evaluating the validity of surrogate 
endpoints in oncology, this method has been criticised as it ignores uncertainty around the treatment effect on the surrogate outcome (which is treated as a fixed covariate in the analysis). Newer methods, such as the bivariate random effects metaanalysis (BRMA) model reported by Bujkiewicz et al., ${ }^{83}$ provides an approach for both the validation and prediction of surrogate endpoints within a Bayesian framework. This approach allows for borrowing of information across studies and fully accounts for all uncertainty surrounding the surrogate relationship. In spite of the generally poor association between response-based outcomes and final outcomes, there may still be instances in which generating predictions on the basis of response is necessary; for example, within health economic models, or more broadly, for decision-making within health technology assessment. In instances where the surrogate association is weak, this uncertainty would manifest as a wider prediction interval. If such predictions are necessary, it is therefore important that all uncertainty is reflected in the model. Future surrogate evaluation studies should consider the use of the BRMA model, rather than conventional meta-regression, as a means of fully reflecting this uncertainty.

\section{CONCLUSIONS}

This systematic review suggests that response-based endpoints such as ORR and CR may not be reliable surrogates for PFS or OS in cancer treatment. Strength of association varied widely between and within studies, with no clear pattern by cancer type. The strength of association between ORR and OS appeared weaker and more variable than that between ORR and PFS, both for associations between absolute endpoints and associations between treatment effects. While there may still be value in using response outcomes as a means of predicting final outcomes such as OS, it is important that those predictions are made on the basis of models which fully reflect the uncertainty around the treatment effect on the surrogate outcome.

\section{ACKNOWLEDGEMENTS}

We thank Andrea Shippam for administrative and endnote support.

\section{AUTHOR CONTRIBUTIONS}

K.C. and P.T. designed the protocol, selected studies, and extracted and analysed data. A.C. designed and undertook the literature searches. K.E. selected studies and extracted data. All authors contributed to the manuscript.

\section{ADDITIONAL INFORMATION}

Ethics approval and consent to participate Ethics approval was not required since all data were already in the public domain.

Data availability All data are provided in the tables, figures and supplementary information

Competing interests The authors declare no competing interests.

Funding information This study was funded by the UK National Institute for Health Research (NIHR) Health Technology Assessment (HTA) Programme, project number NIHR127852.

Supplementary information is available for this paper at https://doi.org/10.1038/ s41416-020-01050-w.

Note This work is published under the standard license to publish agreement. After 12 months the work will become freely available and the license terms will switch to a Creative Commons Attribution 4.0 International (CC BY 4.0).

Publisher's note Springer Nature remains neutral with regard to jurisdictional claims in published maps and institutional affiliations.

\section{REFERENCES}

1. Fleming, T. R. \& Powers, J. H. Biomarkers and surrogate endpoints in clinical trials. Stat. Med. 31, 2973-2984 (2012).

2. Taylor, R. S. \& Elston, J. The use of surrogate outcomes in model-based costeffectiveness analyses: a survey of UK Health Technology Assessment reports. Health Technol. Assess. 13, 1-72 (2009).

3. Fleming, T. R. \& DeMets, D. L. Surrogate end points in clinical trials: are we being misled? Ann. Intern. Med. 125, 605-613 (1996).

4. Prentice, R. L. Surrogate endpoints in clinical trials: definition and operational criteria. Stat. Med. 8, 431-440 (1989).

5. Buyse, M., Molenberghs, G., Burzykowski, T., Renard, D. \& Geys, H. The validation of surrogate endpoints in meta-analyses of randomized experiments. Biostatistics 1, 49-67 (2000).

6. Heller, G. Statistical controversies in clinical research: an initial evaluation of a surrogate end point using a single randomized clinical trial and the Prentice criteria. Ann. Oncol. 26, 2012-2016 (2015).

7. Biomarkers Definitions Working Group. Biomarkers and surrogate endpoints: preferred definitions and conceptual framework. Clin. Pharmacol. Ther. 69, 89-95 (2001).

8. Bucher, H. C., Guyatt, G. H., Cook, D. J., Holbrook, A. \& McAlister, F. A. Users' guides to the medical literature: XIX. Applying clinical trial results. A. How to use an article measuring the effect of an intervention on surrogate end points. JAMA 282, 771-778 (1999)

9. Elston, J. \& Taylor, R. S. Use of surrogate outcomes in cost-effectiveness models: a review of United Kingdom health technology assessment reports. Int. J. Technol. Assess. Health Care 25, 6-13 (2009).

10. German Institute of Quality and Efficiency in Health Care (IQWiG). Validity of surrogate endpoints in oncology (2011).

11. Lassere, M. N., Johnson, K. R., Schiff, M. \& Rees, D. Is blood pressure reduction a valid surrogate endpoint for stroke prevention? An analysis incorporating a systematic review of randomised controlled trials, a by-trial weighted errors-invariables regression, the surrogate threshold effect (STE) and the BiomarkerSurrogacy (BioSurrogate) Evaluation Schema (BSES). BMC Med. Res. Methodol. 12, 27 (2012).

12. Fischer, A., Hernandez-Villafuerte, K., Latimer, N. \& Henshall C. Extrapolation from Progression-Free Survival to Overall Survival in Oncology. Research Papers 001769 (Office of Health Economics, 2016).

13. Davis, S., Tappenden, P. \& Cantrell, A. A Review of Studies Examining the Relationship Between Progression-Free Survival and Overall Survival in Advanced or Metastatic Cancer. University of Sheffield report for NICE Decision Support Unit (2012).

14. Savina, M., Gourgou, S., Italiano, A., Dinart, D., Rondeau, V., Penel, N. et al. Metaanalyses evaluating surrogate endpoints for overall survival in cancer randomized trials: a critical review. Crit. Rev. Oncol. Hematol. 123, 21-41 (2018).

15. Haslam, A., Hey, S. P., Gill, J. \& Prasad, V. A systematic review of trial-level metaanalyses measuring the strength of association between surrogate end-points and overall survival in oncology. Eur. J. Cancer 106, 196-211 (2019).

16. Shea, B. J., Reeves, B. C., Wells, G., Thuku, M., Hamel, C., Moran, J. et al. AMSTAR 2: a critical appraisal tool for systematic reviews that include randomised or non-randomised studies of healthcare interventions, or both. BMJ 358, j4008 (2017).

17. Xie, W., Halabi, S., Tierney, J. F., Sydes, M. R., Collette, L., Dignam, J. J. et al. A systematic review and Recommendation for Reporting of Surrogate Endpoint Evaluation using Meta-analyses (ReSEEM). JNCl Cancer Spectrum 3, https://doi. org/10.1093/jncics/pkz002 (2019).

18. Abdel-Rahman, O. Surrogate end points for overall survival in trials of PD-(L)1 inhibitors for urinary cancers: a systematic review. Immunotherapy 10, 139-148 (2018).

19. Agarwal, N., Bellmunt, J., Maughan, B. L., Boucher, K. M., Choueiri, T. K., Qu, A. Q. et al. Six-month progression-free survival as the primary endpoint to evaluate the activity of new agents as second-line therapy for advanced urothelial carcinoma. Clin. Genitourin. Cancer 12, 130-137 (2014).

20. Agarwal, S. K., Mangal, N., Menon, R. M., Freise, K. J. \& Salem, A. H. Response rates as predictors of overall survival: a meta-analysis of acute myeloid leukemia trials. J. Cancer 8, 1562-1567 (2017).

21. Blumenthal, G. M., Karuri, S. W., Zhang, H., Zhang, L. J., Khozin, S., Kazandjian, D. et al. Overall Response rate, progression-free survival, and overall survival with targeted and standard therapies in advanced non-small-cell lung cancer: US Food and Drug Administration trial-level and patient-level analyses. J. Clin. Oncol. 33, 1008 (2015).

22. Blumenthal, G. M., Zhang, L., Zhang, H., Kazandjian, D., Khozin, S., Tang, S. et al. Milestone analyses of immune checkpoint inhibitors, targeted therapy, and conventional therapy in metastatic non-small cell lung cancer trials: a metaanalysis. JAMA Oncol. 3, e171029 (2017). 
23. Bruzzi, P., Del Mastro, L., Sormani, M. P., Bastholt, L., Danova, M., Focan, C. et al. Objective response to chemotherapy as a potential surrogate end point of survival in metastatic breast cancer patients. J. Clin. Oncol. 23, 5117-5125 (2005).

24. Burzykowski, T., Buyse, M., Piccart-Gebhart, M. J., Sledge, G., Carmichael, J., Luck, $\mathrm{H}$. J. et al. Evaluation of tumor response, disease control, progression-free survival, and time to progression as potential surrogate end points in metastatic breast cancer. J. Clin. Oncol. 26, 1987-1992 (2008).

25. Buyse, M., Thirion, P., Carlson, R. W., Burzykowski, T., Molenberghs, G. \& Piedbois, $P$. Relation between tumour response to first-line chemotherapy and survival in advanced colorectal cancer: a meta-analysis. Lancet 356, 373-378 (2000).

26. Ciani, O., Buyse, M., Garside, R., Peters, J., Saad, E. D., Stein, K. et al. Meta-analyses of randomized controlled trials show suboptimal validity of surrogate outcomes for overall survival in advanced colorectal cancer. J. Clin. Epidemiol. 68, 833-842 (2015).

27. Colloca, G. \& Venturino, A. Trial-level analysis of progression-free survival and response rate as end points of trials of first-line chemotherapy in advanced ovarian cancer. Med. Oncol. 34, 87 (2017).

28. Colloca, G., Venturino, A. \& Guarneri, D. Analysis of response-related and time-toevent endpoints in randomized trials of gemcitabine-based treatment versus gemcitabine alone as first-line treatment of patients with advanced pancreatic cancer. Clin. Colorectal Cancer 15, 264-276 (2016).

29. Colloca, G., Venturino, A. \& Guarneri, D. Analysis of clinical end points of randomised trials including bevacizumab and chemotherapy versus chemotherapy as first-line treatment of metastatic colorectal cancer. Clin. Oncol. 28, e155-e164 (2016).

30. Colloca, G., Vitucci, P. \& Venturino, A. Trial level analysis of prostate-specific antigen-related versus unrelated endpoints in phase III trials of first-line and second-line medical treatments of patients with metastatic castration-resistant prostate cancer. Clin. Genitourin. Cancer 14, 389-397 (2016).

31. Cremolini, C., Antoniotti, C., Pietrantonio, F., Berenato, R., Tampellini, M., Baratelli, C. et al. Surrogate endpoints in second-line trials of targeted agents in metastatic colorectal cancer: a literature-based systematic review and meta-analysis. Cancer Res. Treat. 49, 834-845 (2017).

32. Delea, T. E., Khuu, A., Heng, D. Y. C., Haas, T. \& Soulieres, D. Association between treatment effects on disease progression end points and overall survival in clinical studies of patients with metastatic renal cell carcinoma. Br. J. Cancer 107, 1059-1068 (2012).

33. Elia, E. G., Städler, N., Ciani, O., Taylor, R. S. \& Bujkiewicz S. Combining tumour response and progression free survival as surrogate endpoints for overall survival in advanced colorectal cancer. Cancer Epidemiol. 64, 101665 (2020).

34. Foster, N. R., Qi, Y., Shi, Q., Krook, J. E., Kugler, J. W., Jett, J. R. et al. Tumor response and progression-free survival as potential surrogate endpoints for overall survival in extensive stage small-cell lung cancer: findings on the basis of North Central Cancer Treatment Group trials. Cancer 117, 1262-1271 (2011).

35. Giessen, C., Laubender, R. P., Ankerst, D. P., Stintzing, S., Modest, D. P., Schulz, C. et al. Surrogate endpoints in second-line treatment for mCRC: a systematic literaturebased analysis from 23 randomised trials. Acta Oncol. 54, 187-193 (2015).

36. Hackshaw, A., Knight, A., Barrett-Lee, P. \& Leonard, R. Surrogate markers and survival in women receiving first-line combination anthracycline chemotherapy for advanced breast cancer. Br. J. Cancer 93, 1215-1221 (2005).

37. Hamada, T., Nakai, Y., Isayama, H., Yasunaga, H., Matsui, H., Takahara, N. et al. Progression-free survival as a surrogate for overall survival in first-line chemotherapy for advanced pancreatic cancer. Eur. J. Cancer 65, 11-20 (2016).

38. Han, K., Ren, M., Wick, W., Abrey, L., Das, A., Jin, J. et al. Progression-free survival as a surrogate endpoint for overall survival in glioblastoma: a literature-based metaanalysis from 91 trials. Neuro-Oncology 16, 696-706 (2014).

39. Hashim, M., Pfeiffer, B. M., Bartsch, R., Postma, M. \& Heeg, B. Do surrogate endpoints better correlate with overall survival in studies that did not allow for crossover or reported balanced postprogression treatments? An application in advanced non-small cell lung cancer. Value Health 21, 9-17 (2018).

40. Hotta, K., Kato, Y., Leighl, N., Takigawa, N., Gaafar, R. M., Kayatani, H. et al. Magnitude of the benefit of progression-free survival as a potential surrogate marker in phase 3 trials assessing targeted agents in molecularly selected patients with advanced non-small cell lung cancer: systematic review. PLos ONE 10, e0121211 (2015).

41. Hotta, K., Kiura, K., Fujiwara, Y., Takigawa, N., Oze, I., Ochi, N. et al. Association between incremental gains in the objective response rate and survival improvement in phase III trials of first-line chemotherapy for extensive disease small-cell lung cancer. Ann. Oncol. 20, 829-834 (2009).

42. Ichikawa, W. \& Sasaki, Y. Correlation between tumor response to first-line chemotherapy and prognosis in advanced gastric cancer patients. Ann. Oncol. 17, 1665-1672 (2006).

43. Imaoka, H., Sasaki, M., Takahashi, H., Hashimoto, Y., Ohno, I., Mitsunaga, S. et al. Progression-free survival as a surrogate endpoint in advanced neuroendocrine neoplasms. Endocr. Relat. Cancer 24, 475-483 (2017).
44. Imaoka, H., Sasaki, M., Takahashi, H., Hashimoto, Y., Ohno, I., Mitsunaga, S. et al Alternate endpoints for phase II trials in advanced neuroendocrine tumors. Oncologist 24, 47-53 (2019).

45. Ito, K., Miura, S., Sakaguchi, T., Murotani, K., Horita, N., Akamatsu, H. et al. The impact of high PD-L1 expression on the surrogate endpoints and clinical outcomes of anti-PD-1/PD-L1 antibodies in non-small cell lung cancer. Lung Cancer 128, 113-119 (2019).

46. Johnson, K. R., Ringland, C., Stokes, B. J., Anthony, D. M., Freemantle, N., Irs, A et al. Response rate or time to progression as predictors of survival in trials of metastatic colorectal cancer or non-small-cell lung cancer: a meta-analysis. Lancet Oncol. 7, 741-746 (2006).

47. Kaufman, H. L., Schwartz, L. H., William, W. N. Jr., Sznol, M., Fahrbach, K., Xu, Y. et al. Evaluation of classical clinical endpoints as surrogates for overall survival in patients treated with immune checkpoint blockers: a systematic review and meta-analysis. J. Cancer Res. Clin. Oncol. 144, 2245-2261 (2018).

48. Lee, L., Wang, L. \& Crump, M. Identification of potential surrogate end points in randomized clinical trials of aggressive and indolent non-Hodgkin's lymphoma: correlation of complete response, time-to-event and overall survival end points. Ann. Oncol. 22, 1392-1403 (2011).

49. Li, J., He, Q., Yu, X., Khan, K., Weng, X. W. \& Guan, M. J. Complete response associated with immune checkpoint inhibitors in advanced non-small-cell lung cancer: a meta-analysis of nine randomized controlled trials. Cancer Manag. Res. 11, 1623-1629 (2019)

50. Li, X., Liu, S., Gu, H. \& Wang, D. Surrogate end points for survival in the target treatment of advanced non-small-cell lung cancer with gefitinib or erlotinib. J. Cancer Res. Clin. Oncol. 138, 1963-1969 (2012).

51. Liu, L., Chen, F., Zhao, J. \& Yu, H. Correlation between overall survival and other endpoints in metastatic breast cancer with second- or third-line chemotherapy: literature-based analysis of 24 randomized trials. Bull. Cancer 103, 336-344 (2016).

52. Louvet, C., de Gramont, A., Tournigand, C., Artru, P., Maindrault-Goebel, F. \& Krulik, M. Correlation between progression free survival and response rate in patients with metastatic colorectal carcinoma. Cancer 91, 2033-2038 (2001).

53. Makris, E. A., MacBarb, R., Harvey, D. J. \& Poultsides, G. A. Surrogate end points for overall survival in metastatic, locally advanced, or unresectable pancreatic cancer: a systematic review and meta-analysis of 24 randomized controlled trials. Ann. Surg. Oncol. 24, 2371-2378 (2017).

54. Mangal, N., Salem, A. H., Li, M. Y., Menon, R. \& Freise, K. J. Relationship between response rates and median progression-free survival in non-Hodgkin's lymphoma: a meta-analysis of published clinical trials. Hematol. Oncol. 36, 37-43 (2018).

55. Mangal, N., Salem, A. H., Menon, R. M. \& Freise, K. J. Use of depth of response to predict progression-free survival in relapsed or refractory multiple myeloma: evaluation of results from 102 clinical trials. Hematol. Oncol. 36, 547-553 (2018).

56. Moriwaki, T., Yamamoto, Y., Gosho, M., Kobayashi, M., Sugaya, A., Yamada, T. et al. Correlations of survival with progression-free survival, response rate, and disease control rate in advanced biliary tract cancer: a meta-analysis of randomised trials of first-line chemotherapy. Br. J. Cancer 114, 881-888 (2016).

57. Mushti, S. L., Mulkey, F. \& Sridhara, R. Evaluation of overall response rate and progression-free survival as potential surrogate endpoints for overall survival in immunotherapy trials. Clin. Cancer Res. 24, 2268-2275 (2018).

58. Nakashima, K., Horita, N., Nagai, K., Manabe, S., Murakami, S., Ota, E. et al. Progression-free survival, response rate, and disease control rate as predictors of overall survival in phase III randomized controlled trials evaluating the first-line chemotherapy for advanced, locally advanced, and recurrent non-small cell lung carcinoma. J. Thorac. Oncol. 11, 1574-1585 (2016).

59. Nickolich, M., Babakoohi, S., Fu, P. \& Dowlati, A. Clinical trial design in small cell lung cancer: surrogate end points and statistical evolution. Clin. Lung Cancer 15, 207-212 (2014).

60. Nie, R. C., Chen, F. P., Yuan, S. Q., Luo, Y. S., Chen, S., Chen, Y. M. et al. Evaluation of objective response, disease control and progression-free survival as surrogate end-points for overall survival in anti-programmed death- 1 and antiprogrammed death ligand 1 trials. Eur. J. Cancer 106, 1-11 (2019).

61. Pang, Y., Shen, Z., Sun, J. \& Wang, W. Does the use of targeted agents in advanced gastroesophageal cancer increase complete response? A meta-analysis of 18 randomized controlled trials. Cancer Manag. Res. 10, 5505-5514 (2018).

62. Penel, N., Ryckewaert, T. \& Kramar, A. What is an active regimen in carcinoma of unknown primary sites? Analysis of correlation between activity endpoints reported in phase II trials. Correlation of activity endpoints in phase II trials. Bull. Cancer 101, E19-E24 (2014).

63. Petrelli, F. \& Barni, S. Surrogate end points and postprogression survival in renal cell carcinoma: an analysis of first-line trials with targeted therapies. Clin. Genitourin. Cancer 11, 385-389 (2013).

64. Petrelli, F. \& Barni, S. Surrogate endpoints in metastatic breast cancer treated with targeted therapies: an analysis of the first-line phase III trials. Med. Oncol. 31, 776 (2014). 
65. Ritchie, G., Gasper, H., Man, J., Lord, S., Marschner, I., Friedlander, M. et al. Defining the most appropriate primary end point in phase 2 trials of immune checkpoint inhibitors for advanced solid cancers a systematic review and meta-analysis. JAMA Oncol. 4, 522-528 (2018).

66. Rose, P. G., Tian, C. Q. \& Bookman, M. A. Assessment of tumor response as a surrogate endpoint of survival in recurrent/platinum-resistant ovarian carcinoma: a Gynecologic Oncology Group study. Gynecol. Oncol. 117, 324-329 (2010).

67. Roviello, G., Andre, F., Venturini, S., Pistilli, B., Curigliano, G., Cristofanilli, M. et al. Response rate as a potential surrogate for survival and efficacy in patients treated with novel immune checkpoint inhibitors: a meta-regression of randomised prospective studies. Eur. J. Cancer 86, 257-265 (2017).

68. Sekine, I., Tamura, T., Kunitoh, H., Kubota, K., Shinkai, T., Kamiya, Y. et al. Progressive disease rate as a surrogate endpoint of phase II trials for non-smallcell lung cancer. Ann. Oncol. 10, 731-733 (1999).

69. Shi, Q., Flowers, C. R., Hiddemann, W., Marcus, R., Herold, M., Hagenbeek, A. et al. Thirty-month complete response as a surrogate end point in first-line follicular lymphoma therapy: an individual patient-level analysis of multiple randomized trials. J. Clin. Oncol. 35, 552-560 (2017).

70. Shitara, K., Matsuo, K., Muro, K., Doi, T. \& Ohtsu, A. Correlation between overall survival and other endpoints in clinical trials of second-line chemotherapy for patients with advanced gastric cancer. Gastric Cancer 17, 362-370 (2014).

71. Shukuya, T., Mori, K., Amann, J. M., Bertino, E. M., Otterson, G. A., Shields, P. G. et al. Relationship between overall survival and response or progression-free survival in advanced non-small cell lung cancer patients treated with anti-PD-1/ PD-L1 antibodies. J. Thorac. Oncol. 11, 1927-1939 (2016).

72. Siddiqui, M. K., Tyczynski, J., Pahwa, A. \& Fernandes, A. W. Objective response rate is a possible surrogate endpoint for survival in patients with advanced, recurrent ovarian cancer. Gynecol. Oncol. 146, 44-51 (2017).

73. Sidhu, R., Rong, A. \& Dahlberg, S. Evaluation of progression-free survival as a surrogate endpoint for survival in chemotherapy and targeted agent metastatic colorectal cancer trials. Clin. Cancer Res. 19, 969-976 (2013).

74. Tanaka, K., Kawano, M., Iwasaki, T., Itonaga, I. \& Tsumura H. Surrogacy of intermediate endpoints for overall survival in randomized controlled trials of first-line treatment for advanced soft tissue sarcoma in the pre- and post-pazopanib era: a meta-analytic evaluation. BMC Cancer 19, 56 (2019).

75. Tang, P. A., Bentzen, S. M., Chen, E. X. \& Siu, L. L. Surrogate end points for median overall survival in metastatic colorectal cancer: literature-based analysis from 39 randomized controlled trials of first-line chemotherapy. J. Clin. Oncol. 25, 4562-4568 (2007).

76. Tsujino, K., Kawaguchi, T., Kubo, A., Aono, N., Nakao, K., Koh, Y. et al. Response rate is associated with prolonged survival in patients with advanced non-small cell lung cancer treated with gefitinib or erlotinib. J. Thorac. Oncol. 4, 994-1001 (2009).

77. Tsujino, K., Shiraishi, J., Tsuji, T., Kurata, T., Kawaguchi, T., Kubo, A. et al. Is response rate increment obtained by molecular targeted agents related to survival benefit in the phase III trials of advanced cancer? Ann. Oncol. 21, 1668-1674 (2010).

78. Vidaurre, T., Wilkerson, J., Simon, R., Bates, S. E. \& Fojo, T. Stable disease is not preferentially observed with targeted therapies and as currently defined has limited value in drug development. Cancer J. 15, 366-373 (2009).

79. Wilkerson, J. \& Fojo, T. Progression-free survival is simply a measure of a drug's effect while administered and is not a surrogate for overall survival. Cancer J. 15, 379-385 (2009).

80. Zer, A., Prince, R. M., Amir, E. \& Abdul Razak, A. Evolution of randomized trials in advanced/metastatic soft tissue sarcoma: end point selection, surrogacy, and quality of reporting. J. Clin. Oncol. 34, 1469-1475 (2016).

81. Zhu, R., Lu, D., Chu, Y. W., Chai, A., Green, M., Zhang, N. et al. Assessment of correlation between early and late efficacy endpoints to identify potential surrogacy relationships in non-hodgkin lymphoma: a literature-based meta-analysis of 108 phase II and phase III studies. AAPS J. 19, 669-681 (2017).

82. Buyse, M., Molenberghs, G., Paoletti, X., Oba, K., Alonso, A., Van der Elst, W. et al. Statistical evaluation of surrogate endpoints with examples from cancer clinical trials. Biometrical J. 58, 104-132 (2016).

83. Bujkiewicz, S., Achana, F., Papanikos, T., Riley, R. D. \& Abrams, K. R. Technical Support Document 20: multivariate meta-analysis of summary data for combining treatment effects on correlated outcomes and evaluating surrogate endpoints (2019). http:// nicedsu.org.uk/wp-content/uploads/2019/10/TSD-20-mvmeta-final.pdf. Accessed 28 Aug 2020. 